\title{
Necessidades nos apoios à parentalidade: perceções e práticas dos profissionais de saúde
}

\section{Parenting Support needs: health professional's perceptions and practices}

\author{
Silvana Martins*, Ana Tomás de Almeida* \\ *Universidade do Minho *
}

\begin{abstract}
Resumo
Várias perspetivas teóricas e recomendações internacionais salientam a importância de desenvolver serviços e medidas promotoras de uma parentalidade positiva na comunidade onde vivem as famílias. Conhecendo a realidade portuguesa, verificamos que há uma carência de respostas específicas que promovam as competências parentais, melhorem as relações pais-filhos, o conhecimento, a reflexão, as atitudes, comportamentos e níveis de confiança no papel parental. O presente trabalho tem como propósito apresentar o conceito de parentalidade positiva como um elemento essencial no desenvolvimento de ações psicoeducativas no âmbito dos cuidados de saúde primários e na adoção de práticas profissionais centradas no suporte à parentalidade.

Palavras chave: parentalidade positiva, apoio à parentalidade, cuidados de saúde primários, profissionais de saúde
\end{abstract}

\begin{abstract}
Several theoretical perspectives and international recommendations emphasize the importance of developing services, as well as, and preventive measures that promote positive parenting in the community where families live. Knowing the portuguese reality, we verify that there is a need of specific answers that promote parental skills, improve parent-child relationships, knowledge, reflection, attitudes, behaviours and levels of trust in the parental role. This present study aims to reinforce the concept of positive parenting as an essential element for the construction and development of psychoeducational actions in primary health care and for the implementation of professional practices focused on parenting support. Keywords: positive parenting, parenting support, primary health care, health professionals
\end{abstract}

\section{Introdução}

A família assume-se como um contexto fundamental para a promoção do desenvolvimento e bem-estar infantil. Assim, é através da interação pais-filhos que as crianças começam a construir a sua personalidade, desenvolvendo características que lhes permitem definir-se como crianças e, mais tarde, como adultos.

As transformações sociais (crises económicas, movimentos migratórios, a difusão dos limites entre o público e o privado, a mediatização das relações, a precaridade dos vínculos familiares, laborais; a radicalização religiosa, etc.) vão refletir-se na forma como os pais desenvolvem e exercem o seu papel parental e em mudanças no cenário educativo das famílias.

Perante todas estas transformações sociais, aliadas a um crescente reconhecimento do impacto do exercício da parentalidade no desenvolvimento das crianças e dos jovens maior é o consenso de que o apoio à parentalidade deverá figurar nas políticas sociais dos diferentes países para que se possa ajudar os pais na complexa tarefa de ser pai e mãe numa sociedade em constante mudança (Rodrigo, Almeida \& Reichle, 2015).

Ao olharmos para os cuidados primários de saúde constatamos que estes são oferecidos em instituições de carácter universal, não estigmatizante, onde o objetivo fundamental é a promoção da saúde das pessoas de uma determinada comunidade. Para além disso, são serviços onde assistimos a um acompanhamento contínuo, consistente e precoce das famílias e das suas crianças desde os primeiros anos de vida (Alpert, 1999, Perrin, Sheldrick, McMenamy, Henson, \& Carter, 2014; American Psychological Association, 2009). É este papel ativo de assistência à saúde que lhes confere uma oportunidade privilegiada para intervir junto dos pais no sentido de os apoiar, fornecendo-lhes ferramentas indispensáveis a um exercício mais positivo da parentalidade (Palacios et al., 2014), onde adquirem competências úteis na resposta às necessidades de desenvolvimento dos seus filhos.

Reconhecendo as características destes serviços e a relevância do exercício parental na promoção de um desenvolvimento pleno e saudável das crianças, a Direção de Geral de Saúde (DGS) tem-se dedicado a elaborar guias onde se alerta para a importância dos profissionais de saúde adotarem práticas e desenvolverem ações que possibilitem aos pais 
mobilizar os recursos pessoais e parentais que os ajudarão a exercer o seu papel parental de forma responsiva e capaz de responder às necessidades das crianças. No fundo, constituem-se como linhas orientadoras para a capacitação dos serviços de saúde e dos respetivos profissionais no sentido de desenvolver um trabalho de prevenção e promoção junto das famílias que recorrem aos cuidados de saúde primários.

É este trabalho conjunto entre profissionais de saúde e famílias que constrói um espaço de apoio aos pais onde a promoção do bem-estar familiar ganha uma expressão significativa. Ao mesmo tempo, o trabalho destes profissionais contribui para a redução dos fatores de risco associados a comportamentos de negligência e maltrato, tornando a experiência da parentalidade numa tarefa mais positiva e saudável.

\section{A parentalidade positiva como resposta às necessidades da criança}

Desde 1990 que as reflexões em torno da parentalidade assumem um destaque nas políticas dos Estados Membros da União Europeia. Ao longo dos anos, este interesse tem conduzido a discussões sobre os aspetos relacionados com a família e com o tipo de políticas capazes de permitir aos pais um exercício mais eficaz e positivo da sua parentalidade que, por sua vez, se traduza num desenvolvimento saudável dos seus filhos e na criação de laços familiares coesos.

Como resultado de todas as discussões em torno desta temática, a Recomendação 19 do Comité do Conselho de Ministros (2006) oferece novas visões e orientações, realçando a responsabilidade dos estados membros no desenvolvimento de políticas e ações de apoio às famílias promotoras de um exercício positivo da parentalidade.

Nesta mesma recomendação define-se parentalidade positiva como "o comportamento dos pais fundamentado no superior interesse da criança, que cuida, desenvolve as suas capacidades, não é violento e oferece reconhecimento e orientação, e que incluí o estabelecimento de limites que permitem um desenvolvimento pleno da criança" (Council of Europe, 2006, p.3). No fundo, esta definição vem reforçar a ideia de que o objetivo de ser pai ou mãe passa por promover relações positivas no seio da família, baseadas na responsabilidade parental, onde as crianças vêm garantidos os seus direitos e onde encontram um espaço que lhes permita um desenvolvimento pleno (Rodrigo et al., 2015).

Esta visão do comité europeu realça e valoriza a família como o contexto mais próximo da realidade da criança, reforçando a importância das interações que ela estabelece com os seus pais ou cuidadores ao longo do seu crescimento e que assumem uma ajuda preciosa no momento em que ela precisa de ser resiliente ou de enfrentar situações difíceis (Rutter, 1985, cit. in Sim, Costigan \& Boone, 2011). Para além destes aspetos é amplamente conhecido que as relações positivas entre pais e filhos podem constituir-se como um fator de proteção para as crianças em geral e, principalmente, para as crianças em situação de risco uma vez que potencia a diminuição da possibilidade da ocorrência de abuso e/ou maltrato infantil (Sim, Costigan \& Boone, 2011; Kuronen, 2010).

Ao olharmos para o conceito de parentalidade positiva verificamos que a tarefa parental engloba um conjunto de aspetos chave, como por exemplo o apoio, o afeto, a comunicação, o estabelecimento de rotinas, normas, limites e consequências; que se vão solidificando com o acompanhamento e implicação dos pais na vida quotidiana das crianças. É esta diversidade de ações parentais que permite uma resposta eficaz às necessidades dos filhos e que se traduz em ganhos ao nível do desenvolvimento (Daly, 2015; Rodrigo, Máiquez, Martín \& Rodríguez, 2015;).

Em suma, ao direcionar o trabalho de intervenção com famílias tendo por base a contextualização teórica da parentalidade positiva estamos a desenvolver um modelo de intervenção focado nas forças da família, baseado na prevenção e promoção onde o apoio deverá ser equacionado no sentido de responder às necessidades dos pais no exercício da parentalidade, promovendo as competências parentais, o desenvolvimento de competências pessoais e sociais e favorecer a ativação de redes de apoio formais e informais (Jiménez \& Hidalgo, 2016; MacLeod \& Nelson, 2000)

\section{Os cuidados primários de saúde e o seu papel na promoção da parentalidade positiva}

Perante a preocupação com o bem-estar das famílias e em particular das crianças e jovens que delas fazem parte, o Comité de Ministros do Conselho da Europa (2006) refere que é importante reunir conhecimento acerca das famílias e do papel parental, assim como é necessário criar condições para o uso de técnicas de parentalidade positiva tendo em vista o superior interesse da criança. No fundo, o que se pretende reforçar é a necessidade de desenvolver medidas e políticas que visem oferecer o suporte à parentalidade com o objetivo de promover relações familiares positivas e saudáveis e potenciadores de um desenvolvimento integral das crianças e dos jovens.

É neste sentido que se torna fundamental oferecer aos pais um conjunto de serviços que os apoiem, procurando conferir-lhe um carácter universal para que estejam acessíveis quer a famílias consideradas em risco psicossocial, quer em famílias de baixo risco psicossocial ou sem indicadores de risco

Este carácter universal do apoio à parentalidade poderá ser encontrado ao nível dos serviços da saúde pública. Neste sentido, Sanders e colaboradores (2014, p. 339) abordam a questão da saúde pública em torno da parentalidade definindo-a como "uma abordagem que enfatiza o foco na população geral, recorrendo a uma combinação de intervenções universais e direcionadas, para alcançar mudanças significativas ao nível dos índices populacionais". Esta definição reforça a ideia de que os cuidados de saúde primários conseguem chegar a uma tipologia de pais muito diversificada e que isso poderá ter um impacto significativo na comunidade.

Um modelo de intervenção realizado em contexto de saúde pública oferece aos profissionais a possibilidade de envolver mais pais recorrendo a métodos de 
intervenção muito diferenciados, comparativamente a outros serviços disponíveis em contexto comunitário (Prinz \& Sanders, 2007). Embora nos cuidados de saúde primários os cursos de preparação pré-parto sejam amplamente aceites e disponíveis, estes não são baseados nas necessidades expressadas pelos participantes, mas sim nas mensagens que os próprios profissionais que ministram os cursos acreditam que devem ser transmitidas (Gagnon \& Sandall, 2011). Este foco dos profissionais poderá limitar o tipo de apoio que é oferecido. Assim, quando temos como ideia de partida o apoio às questões da saúde, estamos a dar destaque à prevenção onde a saúde física e os cuidados gerais da criança ganham expressão. Por outro lado, se os profissionais optarem por realizar ações que têm por base a parentalidade positiva, assistiremos a tipologias de acompanhamento que complementam as questões da saúde infantil, das competências e estilos parentais e o desenvolvimento infantil. Contudo, o acesso ao apoio à parentalidade para além do nascimento ainda não é uma norma (Sanders \& Kirby, 2014). Neste sentido, promover o desenvolvimento e implementação de programas ou de ações capazes de responder às necessidades sentidas pelos pais no exercício da sua parentalidade poderá contribuir para uma maior consciencialização social das funções parentais (Sanders, 2012, cit. in Sanders \& Kirby, 2014).

Assim, a implementação de programas nos Centros/Unidades de saúde poderá permitir alcançar mais famílias e, assim contribuir para o desenvolvimento positivo de uma comunidade, reduzindo a taxa de problemas sociais, emocionais e comportamentais em crianças, assim como o maltrato e negligência infantis (Sanders \& Kirby, 2014).

\section{Saúde e parentalidade: perceções e práticas profissionais}

Conhecendo o impacto que as relações pais-filhos têm no desenvolvimento biopsicossocial das crianças e reconhecendo que o exercício da parentalidade é uma tarefa complexa, facilmente verificamos a necessidade de intervir junto das famílias. Atendendo a estes factos, também concluímos que, em algum momento da sua vida, os pais poderão necessitar de ajuda. Desta forma, é importante desenvolver políticas "amigas" das famílias e capacitar os pais oferecendo-lhes um conjunto de ferramentas que os tornem competentes para um desempenho ótimo da sua tarefa parental.

Apesar de não se encontrarem identificações concretas acerca dos serviços de apoio à parentalidade no âmbito dos cuidados de saúde primários, em diferentes relatórios sobre infância, juventude e famílias, publicados pela DGS encontramos referências que deixam transparecer a preocupação acerca da necessidade da criação destes serviços de apoio e o consequente impacto no desenvolvimento saudável de crianças e jovens. Por exemplo, no Relatório de Saúde Infantil e Juvenil - Programa Nacional (Menezes, Sassetti, \& Prazeres, 2012) reconhece-se a importância de oferecer cuidados antecipatórios como fator de promoção da saúde e de prevenção da doença, mais concretamente concedendo a pais, mães e/ou cuidadores um conjunto de conhecimentos necessários e fundamentais ao melhor desempenho na promoção e proteção dos direitos da criança. Partindo deste reconhecimento, elencaram-se um conjunto de objetivos relacionados com a implementação de medidas que apoiem os pais no exercício da parentalidade, dos quais destacamos: a prevenção das perturbações emocionais e do comportamento e a prevenção do maltrato. Para além destes dois objetivos, o relatório refere a necessidade de “...apoiar e estimular o exercício adequado das responsabilidades parentais e promover o bem-estar familiar e em outros ambientes específicos" (Menezes, Sassetti, \& Prazeres, 2012, p. 8).

Por sua vez, o relatório de Promoção de Saúde Mental na Gravidez e Primeira Infância (Cêpeda, Brito \& Heitor, 2005) reconhece a importância da relação estabelecida entre pais e filhos para o desenvolvimento ajustado das crianças. Neste sentido, este relatório faz referência à necessidade de organizar grupos de pais/ futuros pais, orientados por um enfermeiro, um psicólogo ou um técnico de serviço social, para que os pais partilhem experiências, informações e recebam suporte.

Com base nestes relatórios conseguimos perceber que em Portugal há uma preocupação em estabelecer e desenvolver intervenções que apoiem e forneçam suporte às famílias. Logo é importante disseminar de forma mais efetiva medidas que ajudem os pais e mães a desenvolver as suas capacidades contribuindo para promoção do desenvolvimento máximo dos seus filhos.

Tendo em conta todas estas linhas orientadoras, cabe aos profissionais e às instituições de saúde delinear estratégias que operacionalizem os objetivos de intervenção com famílias propostos nos diferentes relatórios.

Assim, é necessário melhorar a apresentação, visibilidade e acessibilidade dos serviços de apoio às famílias para que a comunidade os conheça, se possa familiarizar com o conteúdo das ações e consiga aceder-lhes. É na procura desta melhoria na qualidade de ajuda que se presta às famílias, que desafiamos os profissionais e os serviços a repensar a sua prática profissional de maneira a adequarem-se à nova visão acerca da parentalidade (Rodrigo et al., 2014). Daqui nasce a necessidade de os serviços que visam o apoio às famílias se articularem, desenvolvendo boas práticas baseadas nos pressupostos da parentalidade positiva.

Entende-se por boas práticas como "toda a experiência, guiada por princípios, objetivos e procedimentos apropriados, que geraram resultados positivos, demonstrando a sua eficácia e utilidade num determinado contexto" (Rodrigo et al., 2014, p. 25). No domínio profissional, o conceito de boas práticas "pode referir-se a um contínuo de experiência que vão desde o sentido comum ou o uso tradicional de determinadas formas de fazer ou atuar, até à prática profissional baseada em evidências científicas" (Rodrigo et al., 2014, p. 25).

Olhando mais pormenorizadamente para o trabalho dos profissionais de saúde em contexto de cuidados de saúde primários, a American Psychological Association (2009) refere um conjunto de recomendações que 
devem pautar as práticas destes profissionais no sentido de promover o bem-estar familiar, ao mesmo tempo que assumem um carácter preventivo relativamente ao maltrato. Assim, consideram que os cuidados de saúde primários devem: 1) integrar a promoção de relações seguras, estáveis e estimulantes para as crianças através de relações parentais no acompanhamento relativo à saúde física; 2) promover o acesso universal a programas de parentalidade positiva baseados em evidência e com carácter preventivo a famílias de diversas origens socioeconómicas, culturais, raciais e étnicas; 3) promover a interdisciplinaridade, as interações e os sistemas de colaboração entre instituições e profissionais para implementar medidas de prevenção do maltrato; 4) desenvolver esforços nacionais no âmbito dos cuidados de saúde primários para evitar o maltrato infantil.

Apesar desta visão ser mais direcionada para a prevenção do maltrato na infância, estas práticas englobam os pressupostos enunciados por Rodrigo e colaboradores (2014) relativamente ao conceito de boas práticas e poderão originar ações e ou programas que se adequem a um grupo muito diversificado de famílias.

Desta forma, o estabelecimento de boas práticas nos serviços de apoio à família permite, desde logo, a mobilização do serviço e favorece o aparecimento e difusão de ideias criativas e inovadores. Todo este esforço profissional e aplicação de novas ideias refletir-se-á numa melhoria contínua das instituições de saúde, facilitando o trabalho em rede e criando sinergias de trabalho que respondam de forma efetiva às necessidades das famílias (Rodrigo et al., 2014).

É todo este trabalho profissional conjunto que poderá permitir que as medidas de promoção de competências parentais nos cuidados de saúde primários possam ganhar expressão na intervenção dos profissionais de saúde com as famílias. Consequentemente, este novo olhar sobre a parentalidade permitirá a obtenção de resultados muito positivos no bem-estar geral da família, e das crianças em particular, uma vez que veem satisfeitas as suas necessidades de desenvolvimento.

\section{Metodologia de Investigação}

O estudo empírico junto dos profissionais de saúde terá como objetivos: 1) fazer um levantamento das necessidades sentidas pelos profissionais no apoio e suporte às famílias com filhos com idades entre os 3 meses e os 5 anos; 2) identificar a perceção que têm os profissionais nos cuidados de saúde primários acerca da promoção da parentalidade positiva; 3) identificar as práticas profissionais relacionadas com a promoção da parentalidade positiva.

A resposta a estes objetivos passará pela conjugação das metodologias quantitativa e qualitativa. Assim, numa primeira fase os profissionais de saúde serão convidados a preencher um breve questionário sobre características sociodemográficas e caracterização do serviço onde exercem a sua atividade profissional e o questionário Medida dos Processos de Cuidados para Prestadores de Serviços (Woodside, Rosenbaum, King,
\& King, 2001; tradução portuguesa de Fátima Maia, 2010, Maia, 2012).

O questionário Medida dos Processos de Cuidados para Prestadores de Serviços é um instrumento que permite recolher informações relacionadas com as perceções dos profissionais de saúde relativamente ao apoio prestado, nomeadamente no que respeita às práticas centradas na família. Este instrumento permite estudar as perceções dos profissionais de saúde relativamente a quatro dimensões (subescalas): Evidenciar Sensibilidade Interpessoal, Providenciar Informação de Caráter Geral, Comunicação de Informação de Caráter Específico sobre a Criança e Tratar as Pessoas com Respeito.

De forma a complementar a informação recolhida pelos questionários e a construir uma visão mais pormenorizada das práticas e das perceções dos profissionais, serão realizadas entrevistas recorrendo a um guião previamente construído.

\section{Conclusão}

Conscientes de que a parentalidade não se exerce no vazio, e sim, em diversas ecologias que facilitam ou dificultam o exercício das diferentes responsabilidades a que os pais são chamados a dar resposta, reconhecemos que o suporte e o apoio parental assumem um papel importante na promoção de uma parentalidade mais satisfatória. Esta forma de olhar a parentalidade serve para compreender as necessidades de apoio das famílias qualquer que seja a sua situação (Rodrigo, Amorós, Arranz, Hidalgo, Máiquez, Martín, Martínez \& Ochaita, 2015).

O suporte parental oferece múltiplas vantagens para pais e crianças. Uma dessas vantagens é a possibilidade de aumentar em grande escala a qualidade de vida de um conjunto alargado de cidadãos, ao mesmo tempo que contribui para o progresso e para várias mudanças nas sociedades europeias.

Rodrigo et al. (2015) alertam para que os países que não apostam na criação e manutenção de serviços e recursos de apoio à família numa dada comunidade estão a optar por um caminho arriscado que dificulta os esforços de prevenção e promoção que caracterizam o conceito de parentalidade positiva e que tal acarretará, no futuro, custos desnecessários que advirão da reduzida qualidade de vida e bem-estar das famílias.

Os esforços para melhorar e expandir as intervenções que oferecem suporte aos pais e cuidadores no sentido de lhes oferecer a possibilidade de desenvolver as habilidades, os conhecimentos, qualidades e confiança para que consigam apoiar as crianças num desenvolvimento saudável deveria tornar-se num compromisso de saúde pública mundial (Patterson, Mockford, Barlow, Pyper \& Stewart-Brown, 2002).

No fundo, a promoção de comportamentos parentais positivos deveria ser encarada pelos profissionais de saúde como uma medida de prevenção primária, à semelhança da vacinação, uma vez que poderá ter um grande impacto no bem-estar das famílias e das crianças em particular (American Psychological Association, 2009). Assim, ao adotarem um conjunto de práticas 
profissionais promotoras de competências parentais, os profissionais de saúde estão a permitir que os serviços se desenvolvam com o objetivo de responder, de forma universal, às necessidades das famílias. Todo este investimento em intervenções com $o$ foco na parentalidade positiva é um excelente promotor do bem-estar geral da comunidade onde atuam.

\section{Referências}

American Psychology Association. (2009). Effective strategies to support positive parenting in community health centers: Report of the working group of child maltreatment prevention community health centers. Washington: American Psychology Associations. Retirado

de https://www.apa.org/pi/prevent-violence/resources/po sitive-parenting.pdf.

Alpert, J. J. (1999). History of community pediatrics. Pediatrics, 103(6), 1420-1421.

Cepêda, T., Brito, I., \& Heitor, M. (2005). Promoção da saúde mental na gravidez e primeira infância: manual de orientação para profissionais de saúde. Ministério da Saúde: Direção Geral de Saúde. Acedido a 9 de janeiro de 2016, de: http://www.dgs.pt/accao-de-saude-para-criancas-e-jov ens-em-risco/ficheiros-externos/pub-saude_mental_e_ gravidez_folheto_dgs_2005-pdf.aspx

Council of Europe. (2006). Recommendatios Rec(2006) 19 of the Communittee of Ministers to member states on policy to support positive parenting. Retirado de https://wcd.coe.int/ViewDoc.jsp?id=1073507

Daly, M. (2015). Family and parenting support policy and provision in a global context. Florence: UNICEF Office of Research.

Gagnon, A. J., \& Sandall, J. (2011). Individual or group antenatal education for childbirth or parenthood, or both. Cochrane Database of Systematic Reviews, 11, 4-64.

https://doi.org/10.1002/14651858.CD002869.pub2

Gauthier, A. H. (1999). The sources and methods of comparative family policy research. Comparative Social Research, 18, 31-56.

Jiménez, L., \& Hidalgo, M. V. (2016). La incorporación de prácticas basadas en evidencias en el trabajo con familias: los programas de promoción de parentalidad positiva. Apuntes de Psicología, 34(2-3), 91-100.

Kuronen, M. (Ed.) (2010). Research on families and family polices in Europe: the state of the art. Family Research Centre, University of Jyväskylä

MacLeod, J., \& Nelson, G. (2000). Programs for the promotion of family wellness and the prevention of child maltreatment: A meta-analytic review. Child Abuse and Neglect, 24(9), 1127-1149. https://doi.org/10.1016/S0145-2134(00)00178-2

Maia, F. (2012). Intervenção precoce nas Associações Portuguesas de Paralisia Cerebral: Percepções das famílias, dos profissionais, e dos diretores de serviço. Tese de doutoramento. Instituto de Educação, Universidade do Minho, Braga. Retirado de: file://C:/Users/Utilizador/Documents/Doutoramento/
Maria\%20de\%20Fátima\%20Serdoura\%20Cardoso\%2 0Maia.pdf

Menezes, B., Sassetti, L., \& Prazeres, V. (coord.). (2012). Saúde infantil e juvenil: programa nacional. Direção Geral de Saúde. Retirado de, http://www.dgs.pt/documentos-em-discussao-publica/ consulta-publica-programa-nacional-de-saude-infantil -e-juvenil-jpg.aspx

Patterson, J., Mockford, C., Barlow, J., Pyper, C., \& Stewart-Brown, S. (2002). Need and demand for parenting programmes in general practice. Archives of Diseases in Childhood, 8, 468-471. Doi: 10.1136/adc.87.6.468

Palacios, J., Moreno, M., González, M., \& Oliva, A. (2014). Proyecto Apego sobre evaluación y promoción de competencias parentales en el sistema sanitario público andaluz. Retirado de, http://www.juntadeandalucia.es/salud/export/sites/csal ud/galerias/documentos/p_4_p_2_promocion_de_la_s alud/apego/apego1.pdf

Perrin, E. C., Sheldrick, R. C., McMenamy, J. M., Henson, B. S., \& Carter, A. S. (2014). Improving Parenting Skills for Families of Young Children in Pediatric Settings. Jama Pediatrics, 168(1), 16. https://doi.org/10.1001/jamapediatrics.2013.2919

Prinz, R. J., \& Sanders, M. R. (2007). Adopting a population-level approach to parenting and family support interventions. Clinical Psychology Review, 27(6), 739-749. https://doi.org/10.1016/j.cpr.2007.01.005

Rodrigo, M.J. Máiquez, M.L., Martín, J.C. y Rodríguez, B. (2015). La parentalidad positiva desde la prevención y la promoción. En M.J. Rodrigo (Coord.), Manual práctico de parentalidad positiva (pp. 25-43). Madrid: Síntesis

Rodrigo, M., Almeida, A., \& Reichle, B. (2015). Evidence-based parent education programs: a european perspective. In. J. Ponzetti (Ed.), Evidence-based parenting education: a global perspective (pp. 83-104). New York: Routledge.

Rodrigo, M., Amorós, P., Arranz, E., Hidalgo, M., Máiquez, M., Martín, J., Martínez, R., \& Orchaita, E. (2014). Guía de buenas prácticas en parentalidad positiva: un recurso para apoyar la práctica professional con familias. Federación Española de Municipios y Provincias. Retirado de, http://www.observatoriodelainfancia.es/oia/esp/descar gar.aspx?id=4795\&tipo=documento.

Sanders, M., Kirby, J., Tellegen, C., \& Day, J. (2014). The Triple P-Positive Parenting Program: A systematic review and meta-analysis of a multi-level system of parenting support. Clinical Psychology Review, 34, 337-357.

Sim, A., Costigan, J., \& Boone, L. (2011). Generación de indicios en los que basarse: intervenciones para fortalecer a la familia en contextos humanitarios. Espacio Para la infancia. Fundación Bernad van Leer.

Smith, R. (1997). Parent Education: empowerment or control? Children \& Society, 11, 108-116. 